\title{
Constructible Functions on 2-dimensional Analytic Manifolds
}

\author{
Isabelle BONNARD and Federica PIERONI \\ Lycée Poincaré \\ 2 rue de la Visitation BP 4202 \\ 54042 Nancy Cedex, France \\ isabelle.bonnard@laposte.net \\ Dipartimento di Matematica \\ Via Filippo Buonarroti 2 \\ 56127 Pisa, Italy \\ pieroni@mail.dm.unipi.it
}

Recibido: 17 de Noviembre de 2003

Aceptado: 19 de Febrero de 2004

\begin{abstract}
We present a characterization of sums of signs of global analytic functions on a real analytic manifold $M$ of dimension two. Unlike the algebraic case, obstructions at infinity are not relevant: a function is a sum of signs on $M$ if and only if this is true on each compact subset of $M$. This characterization gives a necessary and sufficient condition for an analytically constructible function, i.e. a linear combination with integer coefficients of Euler characteristic of fibres of proper analytic morphisms, to be such a sum of signs.
\end{abstract}

Key words: global semianalytic sets, constructible functions.

2000 Mathematics Subject Classification: 14P15

\section{Introduction}

Let $M$ be a paracompact real analytic manifold. A function $\varphi: M \rightarrow \mathbb{Z}$ is said to be semianalytically constructible if it is constant on each element of a finite partition of $M$ into global semianalytic sets.

Assume $\operatorname{dim} M=2$. The aim of this paper is to characterize functions $M \rightarrow \mathbb{Z}$ which are (finite) sums of signs of global analytic functions on $M$. This is done in two steps: the reduction to the characterization of functions which are generically sums of signs, and, then, the solution of this generic problem using a characterization of principal open sets (i.e. sets of the form $\{x \in M \mid f(x)>0\}$ where $f$ is a

The authors are partially supported by European Research Training Network HPRN-CT-00271 
global analytic function on $M)$. We show that the obstructions for a semianalytically constructible function $\varphi$ on $M$ to be a sum of signs of global analytic functions lie in the boundaries of the $\varphi^{-1}(n)$ for $n \in \mathbb{Z}$. There are similar results on compact algebraic sets of any dimension, for sums of signs of polynomials ([9]) and sums of signs of Nash functions ([10]). The compactness assumption is not necessary in our situation: $\varphi$ is a sum of signs of global analytic functions on $M$ if and only if it is true on each compact subset of $M$. Compare with [11] for similar results.

Our motivation is to study analytically constructible functions. These functions are defined as linear combinations, with integer coefficients, of Euler characteristic of fibres of proper analytic morphisms. Even if $M$ is compact of dimension two, the two classes of analytically constructible and semianalytically constructible functions are not contained in each other. Our characterization of sums of signs proves that if $\operatorname{dim} M=2$, the intersection between these two classes coincides with the class of sums of signs of global analytic functions on $M$. Similar results are known in the algebraic case, cf. [13], [22], and in the Nash case for a compact set, cf. [10]. We also prove that the compactness assumption is not necessary in dimension two for the Nash case.

The paper is organized as follows. In the first section, we present semianalytically constructible functions, analytically constructible functions, and analogous objects in the algebraic setting. The second section is devoted to a characterization of principal open sets (in any dimension), and a characterization of sums of signs of analytic functions in dimension two. In the third part, we study analytically constructible and Nash constructible functions in dimension two.

We want to thank Francesca Acquistapace, Carlos Andradas, Fabrizio Broglia and Michel Coste for their helpful suggestions.

\section{Constructible functions}

\subsection{The algebraic case}

We first present some known results in the algebraic setting, in order to motivate our work.

If $V \subset \mathbb{R}^{k}$ is an affine algebraic set, a function $\varphi: V \rightarrow \mathbb{Z}$ is semialgebraically constructible if it admits a presentation as a finite sum

$$
\varphi=\sum_{i} m_{i} \mathbf{1}_{X_{i}}
$$

where the $m_{i}$ 's are integers, the $X_{i}$ 's are semialgebraic subsets of $V$ and $\mathbf{1}_{X_{i}}$ denotes the characteristic function of the subset $X_{i}$, cf. [19].

A function $\varphi: V \rightarrow \mathbb{Z}$ is said to be algebraically constructible (respectively Nash constructible) if it can be written as a linear combination with integer coefficients of Euler characteristic of fibres of proper polynomial maps defined on algebraic sets 
(respectively, on connected components of algebraic sets), i.e.

$$
\varphi(x)=\sum_{i=1}^{r} m_{i} \chi\left(f_{i}^{-1}(x)\right) \quad \text { for all } x \in V
$$

where $\chi$ denotes the Euler characteristic, the $m_{i}$ 's are integers, the $f_{i}: Z_{i} \rightarrow V$ are proper polynomial maps and the $Z_{i}$ 's are real algebraic sets (respectively connected components of real algebraic sets).

Algebraically constructible and Nash constructible functions have been introduced by McCrory and Parusiński in [19] to study the topology of real algebraic sets and semialgebraic arc-symmetric sets respectively. See [20] for a survey.

Note that algebraically constructible functions and Nash constructible functions are semialgebraically constructible. This will be one of the main differences with the analytic case.

Algebraically constructible functions coincide with sums of signs of polynomials, see [13] or [22]. If $V$ is compact, Nash constructible functions on $V$ are sums of signs of semialgebraic arc-analytic functions on $V$, cf. [10]. (A function on an analytic set is arc-analytic if it becomes analytic when composed with any analytic arc on the set, cf. [17].)

\subsection{Constructible functions in the analytic setting}

Let $M$ be a paracompact real analytic manifold. We denote by $\mathcal{O}(M)$ the ring of global analytic functions on $M$. If $M$ is connected, we denote by $\mathcal{M}(M)$ the field of meromorphic functions on $M$, i.e. the quotient field of $\mathcal{O}(M)$.

A function $\varphi: M \rightarrow \mathbb{Z}$ is said to be constructible if it is constant on each element of a locally finite partition of $M$ into subanalytic sets, cf. [23], [16].

A subset $S \subset M$ is called global semianalytic if it can be written in the form

$$
S=\bigcup_{i=1}^{p}\left\{x \in M \mid f_{i}(x)=0, g_{i, 1}(x)>0, \ldots, g_{i, r_{i}}(x)>0\right\}
$$

where $f_{i}, g_{i, j} \in \mathcal{O}(M)$. It is said to be basic open if $S=\left\{x \in M \mid g_{1}(x)>\right.$ $\left.0, \ldots, g_{r}(x)>0\right\}$ with $g_{i} \in \mathcal{O}(M)$, and to be principal open if $S=\{x \in M \mid$ $g(x)>0\}$ with $g \in \mathcal{O}(M)$.

Definition 1.1. A function $\varphi: M \rightarrow \mathbb{Z}$ is said to be semianalytically constructible on $M$ if it admits a presentation as a finite sum

$$
\varphi=\sum_{i} m_{i} \mathbf{1}_{X_{i}}
$$

where the $m_{i}$ 's are integers and the $X_{i}$ 's are global semianalytic subsets of $M$. 
In other words, a function $\varphi: M \rightarrow \mathbb{Z}$ is semianalytically constructible if it is constant on each element of a finite partition of $M$ into global semianalytic sets. Note that semianalytically constructible functions are in particular constructible.

Clearly, all the previous definitions also make sense on an analytic subset $X$ of $\mathbb{R}^{k}$.

Let $X \subset \mathbb{R}^{k}$ be a real analytic set, and let $\varphi$ be a constructible function on $X$ with compact support. It admits a presentation (1) where the $X_{i}$ 's are compact subanalytic subsets of $X$. Then, the Euler integral of $\varphi$ on $X$ is defined as

$$
\int_{X} \varphi=\sum_{i} m_{i} \chi\left(X_{i}\right),
$$

cf. [19], [20] and [23]. This integer depends only on $\varphi$.

If $f: Y \rightarrow X$ is a proper analytic map from a real analytic set $Y \subset \mathbb{R}^{l}$, and $\psi$ is constructible on $Y$, the pushforward of $\psi$ by $f$ is the constructible function $f_{*} \psi$ on $X$ defined by

$$
\forall x \in X, f_{*} \psi(x)=\int_{Y} \psi \mathbf{1}_{f^{-1}(x)} .
$$

Definition 1.2. A function $\varphi: X \rightarrow \mathbb{Z}$ is analytically constructible if it admits a presentation as a finite sum

$$
\varphi=\sum m_{i} f_{i *} \mathbf{1}_{Z_{i}}
$$

where the $m_{i}$ 's are integers, the $Z_{i}$ 's are real analytic sets, and each $f_{i}: Z_{i} \rightarrow X$ is a proper analytic map. Then, for $x \in X$, we have $\varphi(x)=\sum m_{i} \chi\left(f_{i}^{-1}(x)\right)$.

Clearly, semialgebraically constructible functions are semianalytically constructible. Algebraically constructible and Nash constructible functions are analytically constructible.

Unlike the algebraic case, the pushforward operation does not preserve semianalytically constructible functions, even if $\operatorname{dim} X=1$. So analytically constructible functions are not in general semianalytically constructible.

Example 1.3. Set $Z=\bigcup_{n>0}\left\{(x, y) \in \mathbb{R}^{2} \mid x-n=y^{2}\right\}$. The set $Z$ is a disjoint union of global analytic subsets of $\mathbb{R}^{2}$, so it is global analytic. Let $\pi: Z \rightarrow \mathbb{R}$ be the projection on the first component. Then, $\pi_{*} \mathbf{1}_{Z}: \mathbb{R} \rightarrow \mathbb{Z}$ is analytically constructible, but not semianalytically constructible since it is not finitely valued. Indeed, for each $n \in \mathbb{N}$, we have $\pi_{*} \mathbf{1}_{Z}(x)=2 n$ for any $\left.x \in\right] n-1, n[$.

Note that if $X$ is compact, a constructible function on $X$ is finitely valued, i.e. it is constant on a finite partition of $X$ into subanalytic sets. If $\operatorname{dim} X=1$, any subanalytic set is semianalytic and any semianalytic set is global. So, any constructible function on a compact analytic curve is semianalytically constructible.

But if $\operatorname{dim} X>1$, the compactness assumption is not sufficient: even if $X$ is compact and smooth, there are analytically constructible functions on $X$ which are not semianalytically constructible. Indeed there are compact semianalytic subsets of $X$ which are not global. 
Example 1.4. Set $Z=\left\{(x, y) \in \mathbb{R}^{2} \mid(x-2)^{2}+y^{2}=1\right\}$ and let $f: Z \rightarrow \mathbb{R}^{2}$ be defined by $f(x, y)=\left(x, \sin \frac{1}{x}\right)$. This is a proper analytic morphism since the circle $Z$ is compact and does not intersect the $y$-axis. The values of the function $\varphi=f_{*} \mathbf{1}_{Z}: \mathbb{R}^{2} \rightarrow \mathbb{Z}$ are:

$$
\varphi(x, y)= \begin{cases}1 & \text { if }(x, y)=(1, \sin 1) \text { or }(x, y)=\left(3, \sin \frac{1}{3}\right) \\ 2 & \text { if } 1<x<3 \text { and } y=\sin \frac{1}{x} \\ 0 & \text { else }\end{cases}
$$

This function is not generically semianalytically constructible since the set $\varphi^{-1}(2)=$ $\left\{(x, y) \in \mathbb{R}^{2} \mid 1<x<3, y=\sin \frac{1}{x}\right\}$ is not global. Indeed, any global analytic function on $\mathbb{R}^{2}$ vanishing on $\varphi^{-1}(2)$ has to be identically zero. So the Zariski closure of $\varphi^{-1}(2)$, i.e. the smallest global analytic subset of $\mathbb{R}^{2}$ containing $\varphi^{-1}(2)$, coincides with $\mathbb{R}^{2}$.

Let $g: \mathbb{R}^{2} \rightarrow S^{2}$ be the one-point compactification. Then $(g \circ f)_{*} \mathbf{1}_{Z}: S^{2} \rightarrow \mathbb{Z}$ is an analytically constructible function on a two-dimensional compact analytic manifold which is not semianalytically constructible.

Our aim is to compare analytically constructible functions and sums of signs of analytic functions. If $f$ is a function on $X$, we denote by $\operatorname{sign} f: X \rightarrow\{-1,0,1\}$ the sign of the function $f$. Since $\operatorname{sign} f=\mathbf{1}_{\{f>0\}}-\mathbf{1}_{\{f<0\}}$, it is semianalytically constructible.

Proposition 1.5. Let $X \subset \mathbb{R}^{k}$ be a real analytic set. $A$ sum of signs of analytic functions on $X$ is analytically constructible.

Proof. It is enough to prove that if $f: X \rightarrow \mathbb{R}$ is analytic, then $\operatorname{sign} f$ is analytically constructible. Denote $Y=\left\{(x, t) \in X \times \mathbb{R} \mid f(x)=t^{2}\right\} \subset \mathbb{R}^{k} \times \mathbb{R}$, and let $\pi: Y \rightarrow X$ be the projection on the first component. Then we have sign $f=\pi_{*} \mathbf{1}_{Y}-\mathbf{1}_{X}$, and $\operatorname{sign} f$ is analytically constructible.

Notation 1.6. If $f_{1}, \ldots, f_{k}$ are analytic functions on $X$, we denote by $\left\langle f_{1}, \ldots, f_{k}\right\rangle$ the function $\operatorname{sign} f_{1}+\cdots+\operatorname{sign} f_{k}: X \rightarrow \mathbb{Z}$.

The following remark was kindly suggested by K. Kurdyka and A. Parusiński.

Remark 1.7. Assume the dimension of $X$ is two. Then a sum of signs of subanalytic arc-analytic functions on $X$ is analytically constructible.

Indeed, consider $f: X \rightarrow \mathbb{R}$ a subanalytic arc-analytic function. Let $\pi^{\prime}: X^{\prime} \rightarrow X$ be a desingularization of $X$, and let $f^{\prime}=f \circ \pi^{\prime}: X^{\prime} \rightarrow \mathbb{R}$. By [8, Th. 1.4] or [21, Th. 3.1] there exists a locally finite covering $\left\{\pi_{j}: X_{j} \rightarrow X^{\prime}\right\}$ of $X^{\prime}$ such that each $\pi_{j}$ is a composite of finitely many blowings-up of points, and each $f^{\prime} \circ \pi_{j}$ is analytic. Since points are global analytic subsets of $X^{\prime}$, each $\pi_{j}$ can be seen as a global map. Arguing as in [18] we can "glue" the $\pi_{j}$ 's together: there exists $\pi: \widetilde{X} \rightarrow X^{\prime}$ which is the composite of a locally finite sequence of blowings-up of points and such that 
$f^{\prime} \circ \pi=f \circ \pi^{\prime} \circ \pi$ is analytic. (We cannot repeat this argument in higher dimension: in this case each $\pi_{j}$ given by [8] or [21] is still the composite of finitely many blowings-up with smooth centers, but in general these centers are not global analytic in $X^{\prime}$.)

Let $\Sigma \subset X$ be a global analytic subset such that $\sigma=\pi^{\prime} \circ \pi: X^{\prime} \backslash \sigma^{-1}(\Sigma) \rightarrow X \backslash \Sigma$ is an analytic isomorphism. The restriction of $f$ to $\Sigma$ is analytic by [17]. Since $\Sigma$ is a curve it is coherent and it follows from Cartan's Theorem B that there is an analytic function $g$ on $X$ such that $f_{\mid \Sigma}=g_{\mid \Sigma}$. Then we have sign $f=\left(1-\mathbf{1}_{\Sigma}\right) \sigma_{*} \operatorname{sign}(f \circ \sigma)+$ $\mathbf{1}_{\Sigma} \operatorname{sign} g$, so $\operatorname{sign} f$ is analytically constructible.

\section{Sum of signs and principal sets}

In the first subsection we reduce the problem of characterizing sums of signs of analytic functions in dimension two to the same generic problem. In the second subsection we present a characterization of principal global semianalytic sets (in any dimension), that we use in the third subsection to characterize generic sums of signs of analytic functions in dimension two.

\subsection{From generic sum of signs to sum of signs}

Let $M$ be a paracompact real analytic manifold. We say that a property is true generically on $M$ if it is true on $M$ outside a global analytic subset of $M$ of strictly smaller dimension. We write $=_{\text {gen }}$ for an equality holding generically.

A sum of signs of global analytic functions on $M$ has to be generically constant mod 2 on each connected component of $M$ (since the sign of a global analytic function is so). In dimension one, this condition is sufficient for a semianalytically constructible function to be a sum of signs of global analytic functions. More precisely:

Proposition 2.1. Let $W \subset M$ be an analytic set of dimension one, and let $\varphi$ : $W \rightarrow \mathbb{Z}$ be a semianalytically constructible function. Then $\varphi$ is a sum of signs of global analytic functions on $W$ if and only if $\varphi$ is generically constant mod 2 on each irreducible component of $W$. In particular, $\varphi$ is a sum of signs on $W$ if and only if this is true generically on $W$.

Proof. Note that "generically on $W$ " means "outside a discrete subset of $W$." The necessary condition is clear. For the sufficient condition, write $\varphi={ }_{g e n} \mathbf{1}_{W^{\prime}}+\sum_{i=1}^{s} \varphi_{i}$ where $W^{\prime}$ is a union of irreducible components of $W$ and each $\varphi_{i}$ is a semianalytically constructible function on $W$ taking only the values 1 and -1 . By [15, Prop. 4.4], any semianalytic subset of $W$ is generically global principal. So each $\varphi_{i}$ is generically the sign of an element $g_{i} \in \mathcal{O}(W)$. Let $g \in \mathcal{O}(W)$ be a positive equation of $W^{\prime}$. We have $\varphi={ }_{\text {gen }}\left\langle 1,-g, g_{1}, \ldots, g_{s}\right\rangle$ on $W$, so $\varphi-\left\langle 1,-g, g_{1}, \ldots, g_{s}\right\rangle=k_{1} \mathbf{1}_{D_{1}}+\cdots+k_{m} \mathbf{1}_{D_{m}}$ where $k_{1}, \ldots, k_{m}$ are integers, and $D_{1}, \ldots, D_{m}$ are discrete subsets of $W$. For $l=$ $1, \ldots, m$, let $h_{l} \in \mathcal{O}(W)$ be a positive equation of $D_{l}$. We get $\varphi=\left\langle 1,-g, g_{1}, \ldots, g_{s}\right\rangle+$ $k_{1}\left\langle 1,-h_{1}\right\rangle+\ldots+k_{m}\left\langle 1,-h_{m}\right\rangle$. 
Remark 2.2. Since $W$ is coherent, $\varphi$ is the restriction to $W$ of a sum of signs of global analytic functions on $M$ if and only if it is a sum of signs of global analytic functions on $W$.

If $\operatorname{dim} M \geq 2$, there are semianalytically constructible functions which are sums of signs of global analytic functions only generically. The next proposition compares sums of signs and generic sums of signs in dimension two.

Let $M$ be of any dimension. If $S \subset M$ is a semianalytic set, we denote by $\partial_{Z} S$ the Zariski closure of the Euclidean boundary $\partial S$ of $S$. Assume $S$ is global. If $\operatorname{dim} M \leq 2$ then $\partial S$ is global semianalytic by [12], but this result is not known if $\operatorname{dim} M>2$. In any dimension, $\partial S$ is contained in the union of the zero sets of the functions appearing in a description of $S$, so the codimension of the set $\partial_{Z} S$ is at least one.

Proposition 2.3. Assume $\operatorname{dim} M=2$. Let $\varphi: M \rightarrow \mathbb{Z}$ be a semianalytically constructible function, which is generically a sum of signs of global analytic functions on M. Denote $Y=\bigcup_{n \in \mathbb{Z}} \partial_{Z}\left(\varphi^{-1}(n)\right)$ (in fact the union is finite).

Then $\varphi$ is a sum of signs of global analytic functions everywhere on $M$ if and only if $\varphi$ is generically constant $\bmod 2$ on each global irreducible component of dimension one of $Y$.

Proof. The "only if" part is clear. For the "if" part, let $f_{1}, \ldots, f_{k} \in \mathcal{O}(M)$ be such that $\varphi_{\mid M \backslash W}=\left\langle f_{1}, \ldots, f_{k}\right\rangle_{\mid M \backslash W}$, where $W \subset M$ is an analytic set of dimension one. We can always suppose $\left\langle f_{1}, \ldots, f_{k}\right\rangle_{\mid W}=0$. The function $\varphi$ is generically constant mod 2 on each irreducible component of dimension one of $W$. Indeed, if $W_{1}$ is such a component which is not contained in $Y$, then $W_{1} \cap Y$ is a discrete subset of $W_{1}$. Outside this subset the parity of $\varphi_{\left.\right|_{W_{1}}}$ is the generic parity of $\varphi$ on the connected component of $M$ containing $W_{1}$. By Proposition 2.1 and Remark 2.2 there exist $s_{1}, \ldots, s_{r} \in \mathcal{O}(M)$ such that $\varphi_{\mid W}=\left\langle s_{1}, \ldots, s_{r}\right\rangle_{\mid W}$. Let $t \in \mathcal{O}(M)$ be a positive equation for $W$. Then $\varphi=\left\langle f_{1}, \ldots, f_{k}\right\rangle+\langle 1,-t\rangle \times\left\langle s_{1}, \ldots, s_{r}\right\rangle$.

\subsection{A criterion for principality}

Let $M$ be a paracompact real analytic manifold of dimension $n$, and let $S \subset M$ be a global semianalytic subset. Denote $S^{*}=\operatorname{Int}(\operatorname{Adh}(S))$. This is a semianalytic set, although its global character is only known in dimensions one and two by [12]. Note that $\partial\left(S^{*}\right) \subset \partial S$, so $\partial_{Z}\left(S^{*}\right)$ has codimension at least one in $M$. We define a wall of $S$ as an irreducible component of $\partial_{Z}\left(S^{*}\right)$ of codimension one in $M$. The notion of wall is used in [4], [1], [2] and [11] to study basicness, separation and distribution of signs on algebraic and analytic sets.

Definition 2.4. A wall $W$ of $S$ is even if $\operatorname{dim}\left(W \cap S^{*}\right)=n-1$ or $\operatorname{dim}\left(W \cap(M \backslash S)^{*}\right)=$ $n-1$, and it is odd if $\operatorname{dim}\left(W \cap \operatorname{Adh}\left(S^{*}\right) \cap \operatorname{Adh}\left((M \backslash S)^{*}\right)\right)=n-1$, cf. [1], [3]. (The dimension of a semianalytic set $T$ is the maximum integer $d$ such that $T$ contains regular points of dimension $d$.) 
Proposition 2.5. Let $S \subset M$ be a global semianalytic set. Then $S \backslash \partial_{Z} S$ is principal open if and only if no wall of $S$ is simultaneously odd and even.

Proof. Assume $S \backslash \partial_{Z} S$ is principal open, i.e. there exists $f \in \mathcal{O}(M)$ such that $S \backslash$ $\partial_{Z} S=\{f>0\}$. If a wall $W$ of $S$ is odd then the sign of $f$ changes crossing $W$, and if $W$ is even then the sign of $f$ does not change crossing $W$. So $W$ cannot be simultaneously odd and even.

Since $S \backslash \partial_{Z} S$ is principal open if and only if it is principal open in each component of $M$, it is enough to prove the converse implication if $M$ is connected. Assume that no wall of $S$ is simultaneously odd and even. We build $f \in \mathcal{O}(M)$ such that $S \backslash \partial_{Z} S=\{f>0\}$ and $\partial_{Z} S=\{f=0\}$. Let $Y$ be the union of the odd walls of $S$, and let $p \in \mathcal{O}(M)$ be a positive equation for $\partial_{Z} S$. Denote by $\mathcal{J}$ the ideal sheaf induced by $\mathcal{J}(Y)$. It is enough to prove that $\mathcal{J}$ is principal. Indeed if $g \in \mathcal{O}(M)$ generates $\mathcal{J}(Y)$, then, by construction we get $S \backslash \partial_{Z} S=\{p g>0\}$ or $S \backslash \partial_{Z} S=\{p g<0\}$.

By [11, Lemma 1.2] the ideal sheaf $\mathcal{J}$ is locally principal, so for each $x \in M$ there exists a generator $h_{x}$ of $\mathcal{J}_{x}$. Fix $x \in M$. The definition of odd and even walls still makes sense for the analytic components of the analytic set germ induced by $\partial_{Z} S$ at $x$, and the odd walls of $S_{x}$ are the components of $Y_{x}$. So the analytic function germ $h_{x} p_{x}$ is such that $\left(S \backslash \partial_{Z} S\right)_{x}=\left\{h_{x} p_{x}>0\right\}$ or $\left(S \backslash \partial_{Z} S\right)_{x}=\left\{h_{x} p_{x}<0\right\}$. Changing $h_{x}$ into $-h_{x}$ if necessary, we can assume that $\left(S \backslash \partial_{Z} S\right)_{x}=\left\{h_{x} p_{x}>0\right\}$. We obtain local generators $\left\{h_{i}\right\}$ for $\mathcal{J}$ on a locally finite open covering $\left\{U_{i}\right\}$ of $M$ such that $h_{i_{\mid U_{i} \cap U_{j}}}$ and $h_{j_{\mid U_{i} \cap U_{j}}}$ have the same sign, i.e. the cocycle $\left\{h_{i} / h_{j}\right\}$ is zero in $H^{1}\left(M, \mathbb{Z}_{2}\right)$.

We use now the general theory of sheaves. The exponential map and the associated usual exact sequence

$$
0 \rightarrow \mathcal{O}_{M} \rightarrow \mathcal{O}_{M}^{*} \rightarrow \mathcal{O}_{M}^{*} / \mathcal{O}_{M}^{+}=\mathbb{Z}_{2} \rightarrow 0
$$

induce an isomorphism between $H^{1}\left(M, \mathbb{Z}_{2}\right)$ and $H^{1}\left(M, \mathcal{O}_{M}^{*}\right)$, so the cocycle $\left\{h_{i} / h_{j}\right\}$ is zero also in $H^{1}\left(M, \mathcal{O}_{M}^{*}\right)$. By the exact sequence

$$
0 \rightarrow \mathcal{O}_{M}^{*} \rightarrow \mathcal{M}_{M}^{*} \rightarrow \mathcal{M}_{M}^{*} / \mathcal{O}_{M}^{*} \rightarrow 0
$$

the global section $x \mapsto h_{i, x}$ of the sheaf of the divisors lies in the image of the map $\Gamma\left(M, \mathcal{M}_{M}^{*}\right) \rightarrow \Gamma\left(M, \mathcal{M}_{M}^{*} / \mathcal{O}_{M}^{*}\right)$. This means that there exists a non zero global meromorphic function $g$ on $M$ such that $g_{x} \mathcal{O}^{*}=h_{i, x} \mathcal{O}^{*}$. In particular, $g$ lies in $\mathcal{O}(M)$ since $g_{x}$ lies in $\mathcal{O}_{M, x}$ for each $x \in M$, and $g$ generates $\mathcal{J}(Y)$. The proof is done.

Remark 2.6. Proposition 2.5 proves in particular that $S$ is generically principal if and only if $S \backslash \partial_{Z} S$ is principal open. It also shows that $S$ is generically principal if and only if for each compact set $K \subset M$, the set $S \cap K$ is generically principal in $K$. See [11] for similar results. 


\subsection{Generic sums of signs in dimension two}

Let $M$ be a paracompact real analytic manifold of dimension two, and let $\varphi: M \rightarrow \mathbb{Z}$ be a semianalytically constructible function. To know if $\varphi$ is a sum of signs of global analytic functions, by Proposition 2.3 it is enough to determine if $\varphi$ is generically such a sum of signs.

Lemma 2.7. If $\varphi$ is generically constant $\bmod 4$, then $\varphi$ is generically a sum of signs of global analytic functions on $M$.

Proof. It is enough to prove that if $S \subset M$ is a global semianalytic subset of $M$, then $4 \mathbf{1}_{S}$ is generically a sum of signs of global analytic functions on $M$. By [15], the set $S$ is generically the union of two basic open global semianalytic sets $S_{1}$ and $S_{2}$, so $\mathbf{1}_{S}=$ gen $\mathbf{1}_{S_{1}}+\mathbf{1}_{S_{2}}-\mathbf{1}_{S_{1} \cap S_{2}}$. Since the intersection of two basic open sets is still basic open, we can assume that $S$ is basic open.

The stability index of an analytic manifold is defined as the smallest integer $s$ such that every basic open set is an intersection of at most $s$ principal open sets if such an integer exists, and as $\infty$ otherwise. By [15], the stability index of $M$ is two. So there exist $f_{1}, f_{2} \in \mathcal{O}(M)$ such that $S=\left\{x \in M \mid f_{1}(x)>0, f_{2}(x)>0\right\}$ and we have $4 \mathbf{1}_{S}={ }_{\text {gen }}\left\langle 1, f_{1}, f_{2}, f_{1} f_{2}\right\rangle$.

Remark 2.8. It is not known if, in dimension $n>2$, a semianalytically constructible function which is generically constant mod $2^{n}$ is generically a sum of signs of analytic functions. Indeed, the previous proof cannot be repeated since the value of the stability index of an analytic manifold of dimension $>2$ is not known.

A wall of the function $\varphi$ is defined as a wall of a $\varphi^{-1}(n)$ for some $n \in \mathbb{Z}$. If $W$ is such a wall, we define the function $\partial_{W} \varphi: W \rightarrow \mathbb{Z}[1 / 2]$ in the following way. Let $x \in W$. If $x \in \operatorname{Sing}\left(\bigcup_{n} \partial_{Z} \varphi^{-1}(n)\right)$, we set $\partial_{W} \varphi(x)=0$. Otherwise $\varphi$ is constant on each of the two connected components of the germ $(M \backslash W)_{x}$, and $\partial_{W} \varphi(x)$ is defined as the average of the values of $\varphi$ on these two components.

The function $\partial_{W} \varphi$ is constant on each element of a finite global semianalytic partition of $W$, but a priori it is not integer-valued. If $\varphi$ is generically constant $\bmod 2$ on each connected component of $M$, then $\partial_{W} \varphi$ is semianalytically constructible.

In the algebraic or Nash context, the functions $\partial_{W} \varphi$ have been used to study algebraically constructible and Nash constructible functions. (cf. [9], [10]).

Remark 2.9. If $M$ is embedded in some $\mathbb{R}^{k}$, and $\varphi$ is a semianalytically constructible function on $M$, the link of $\varphi$ is the constructible function $\Lambda \varphi$ on $M$ defined by

$$
\Lambda \varphi(x)=\int_{M} \varphi \mathbf{1}_{S(x, \varepsilon)}
$$

where $\varepsilon>0$ is small enough, and $S(x, \varepsilon)$ denotes the sphere with center $x$ and radius $\varepsilon$ in $M$, cf. [19]. This value depends only on $\varphi$ and $x$. It can be checked that $\partial_{W} \varphi=g_{\text {gen }}\left(\varphi-\frac{1}{2} \Lambda \varphi\right)_{\mid W}$ for any wall $W$ of $\varphi$. 
Theorem 2.10. Let $M$ be a paracompact real analytic manifold of dimension two. A semianalytically constructible function $\varphi: M \rightarrow \mathbb{Z}$ is generically a sum of signs of global analytic functions if and only if, for each wall $W$ of $\varphi$, the function $\partial_{W} \varphi$ is integer-valued and generically constant $\bmod 2$ on $W$.

Proof. Assume there exist $f_{1}, \ldots, f_{r} \in \mathcal{O}(M)$ such that $\varphi=$ gen $\left\langle f_{1}, \ldots, f_{r}\right\rangle$ on $M$. Let $W$ be a wall and let $t \in \mathcal{O}(M)$ be an uniformizer of $o f(M)_{\mathcal{J}}(W)$, which is a discrete valuation ring of rank one by [11, Prop.1.5]. For $i=1, \ldots, r$, write $f_{i}=t^{k_{i}} g_{i}$ where $k_{i}$ is an integer and $g_{i} \in \mathcal{O}(M)$ is not identically zero on $W$. Then on $W$ we have

$$
\partial_{W} \varphi=\text { gen } \sum_{i \mid k_{i} \text { even }} \operatorname{sign} g_{i}
$$

To prove the converse implication, assume that on each wall $W$ of $\varphi$, the function $\partial_{W} \varphi: W \rightarrow \mathbb{Z}$ is generically integer-valued and generically constant $\bmod 2$. We first prove that $\varphi$ is generically constant $\bmod 2$ on each connected component of $M$. Let $\bar{\varphi}: M \rightarrow\{0,1\}$ be a semianalytically constructible function such that $\varphi-\bar{\varphi}$ is generically even. If $\bar{\varphi}$ had a wall $W$, then $\partial_{W} \bar{\varphi}$ would be generically $1 / 2$ on a non empty open subset of $W$, and $\partial_{W} \varphi={ }_{\text {gen }} \partial_{W}(\varphi-\bar{\varphi})+\partial_{W} \bar{\varphi}$ would not be integervalued on $W$. So $\bar{\varphi}$ has no wall. On each connected component of $M$, the function $\bar{\varphi}$ is generically constant and $\varphi$ is generically constant $\bmod 2$. Write $\varphi=\varphi_{1}+\varphi_{2}+\mathbf{1}_{C}$ where $\varphi_{1}, \varphi_{2}: M \rightarrow \mathbb{Z}$ are semianalytically constructible functions such that $\varphi_{1}$ is generically constant $\bmod 4, \varphi_{2}$ takes only the two values 1 and -1 and $C$ is a union of connected components of $M$.

By Lemma 2.7, it is enough to prove that $\varphi_{2}$ is generically a sum of signs of analytic functions. Denote $S=\varphi_{2}^{-1}(1)$ and let $W$ be a wall of $S$. It is also a wall of $\varphi$. Note that $\partial_{W} \varphi_{2}=$ gen 0 on $W \cap \operatorname{Adh}\left(S^{*}\right) \cap \operatorname{Adh}\left((M \backslash S)^{*}\right)$, and $\partial_{W} \varphi_{2}=$ gen 1 (respectively -1) on $W \cap S^{*}$ (respectively on $\left.W \cap(M \backslash S)^{*}\right)$. As we have $\partial_{W} \varphi_{2}={ }_{g e n} \partial_{W} \varphi-\partial_{W} \varphi_{1}$ if $W \not \subset C$ and $\partial_{W} \varphi_{2}={ }_{g e n} \partial_{W} \varphi-\partial_{W} \varphi_{1}-1$ if $W \subset C$, the function $\partial_{W} \varphi_{2}$ is generically constant mod 2 on $W$. So the wall $W$ of $S$ cannot be simultaneously odd and even, and by Proposition 2.5 there exists $f \in \mathcal{O}(M)$ such that $S=$ gen $\{f>0\}$. Then $\varphi_{2}=$ gen $\operatorname{sign} f$ and the proof is done.

Corollary 2.11. Let $M$ be a paracompact real analytic manifold of dimension two. A semianalytically constructible function $\varphi: M \rightarrow \mathbb{Z}$ is a sum of signs of global analytic functions if and only if, for each compact set $K \subset M$, the function $\varphi_{\left.\right|_{K}}$ is the restriction to $K$ of a finite sum of signs of global analytic functions on $M$.

Remark 2.12. The problem of characterizing sums of signs, i.e. signatures of quadratic forms, was studied also for constructible functions on the real spectrum of a field $K$. It was solved by Becker and Bröcker in terms of particular finite spaces of orderings, namely of fans (cf. [7]). If $K$ is the field of rational functions on an irreducible real algebraic set $V$, the so called Artin-Lang property acts as a translator between constructible subsets of the real spectrum of $K$ and semialgebraic subsets of $V$. It allows 
one to read statements on the real spectrum as geometric results (cf. [9], [4], [1]). If $M$ is an analytic manifold, the Artin-Lang property, which fails for $\operatorname{Spec}_{\mathrm{r}} \mathcal{O}(M)$ and is known for $\operatorname{Spec}_{\mathrm{r}} \mathcal{M}(M)$ only if $\operatorname{dim} M \leq 2$, is not such a good tool for studying geometric problems. One of the reasons is the presence of unbounded orderings, i.e. orderings whose associated ultrafilter does not specialize to a point (cf. [5]). Using Theorem 2.10 and arguing as in [11], it can be shown that if $\operatorname{dim} M \leq 2$, unbounded orderings are not relevant to recognize signatures of quadratic forms among constructible functions $\operatorname{Spec}_{\mathrm{r}} \mathcal{M}(M) \rightarrow \mathbb{Z}$. More precisely:

Let $M$ be a paracompact connected real analytic manifold of dimension two and let $\widetilde{\varphi}: \operatorname{Spec}_{\mathrm{r}} \mathcal{M}(M) \rightarrow \mathbb{Z}$ be a constructible function. The function $\widetilde{\varphi}$ is the signature of a quadratic form if and only if for each fan $F$ of $\operatorname{Spec}_{\mathrm{r}} \mathcal{M}(M)$ made of bounded orderings, we have $\sum_{\sigma \in F} \widetilde{\varphi}(\sigma) \equiv 0$ $\bmod |F|$.

\section{Analytically constructible functions in dimension two}

\subsection{The analytic case}

Let $X$ be a real analytic subset of $\mathbb{R}^{k}$, and let $\varphi$ be an analytically constructible function on $X$ (cf. Definition 1.2). As remarked in Section 1, the function $\varphi$ is not necessarily semianalytically constructible, even if $\operatorname{dim} X=1$. Conversely semianalytically constructible functions are not in general analytically constructible. For instance, analytically constructible functions must verify some parity conditions, cf. Lemma 3.1. The aim of this subsection is to prove that, if $M$ is a real analytic manifold of dimension $\leq 2$, the intersection between the classes of analytically constructible functions and semianalytically constructible functions coincides with the class of sums of signs of analytic functions on $M$.

Using techniques of desingularization of analytic sets and [19, Prop.5.1], [19, Th.2.5], we get the following lemma:

Lemma 3.1. Let $X \subset \mathbb{R}^{k}$ be an irreducible analytic set, and let $\varphi$ be an analytically constructible function on $X$.

(i) The function $\varphi$ is constant $\bmod 2$ on $X$ outside a subanalytic subset of $X$ of strictly lower dimension.

(ii) The function $\frac{1}{2} \Lambda \varphi$ is analytically constructible on $X$.

Proposition 3.2. Let $X \subset \mathbb{R}^{k}$ be an analytic curve. Let $\varphi$ be a semianalytically constructible function on $X$, i.e. a finitely valued constructible function on $X$. The function $\varphi$ is analytically constructible if and only if it is a sum of signs of global analytic functions on $X$. 
Proof. The sufficient condition is given by Proposition 1.5. Conversely, if $\varphi$ is analytically constructible, then by the first point of Lemma 3.1, it is generically constant mod 2 on each irreducible component of $X$ of dimension one (since a subanalytic set of dimension 0 is a discrete set of points). By Proposition 2.1, it is a sum of signs of global analytic functions on $X$.

Theorem 3.3. Let $M$ be a paracompact real analytic manifold of dimension two. A semianalytically constructible function $\varphi: M \rightarrow \mathbb{Z}$ is analytically constructible if and only if it is a sum of signs of global analytic functions on $M$.

Proof. The "if" part is given by Proposition 1.5. For the "only if" part, assume that $\varphi$ is analytically constructible. Let $W$ be a wall of $\varphi$. By Remark 2.9 and Lemma 3.1, the function $\partial_{W} \varphi$ is generically analytically constructible on $W$. In particular, $\partial_{W} \varphi$ is integer-valued and generically constant $\bmod 2$ on $W$. By Theorem 2.10, the function $\varphi$ is generically a sum of signs of global analytic functions on $M$.

Let $W^{\prime}$ be an irreducible component of dimension one of

$$
\bigcup_{n \in \mathbb{Z}} \partial_{Z}\left(\varphi^{-1}(n)\right)
$$

The function $\varphi_{\left.\right|_{W^{\prime}}}$ is analytically constructible on $W^{\prime}$, so it is generically constant mod 2 on $W^{\prime}$. Using Proposition 2.3, we conclude that $\varphi$ is a sum of signs of global analytic functions on $M$.

The result of Theorem 3.3 is no more true if $\operatorname{dim} M \geq 3$, or if we work on a singular analytic set, cf. Remark 3.5 in [10]. On a normal surface, using Remark 1.7 and [12], we get the following:

Corollary 3.4. Let $X$ be a normal real analytic surface. The intersection of the class of analytically constructible functions and the class of semianalytically constructible functions is exactly the class of sums of signs of subanalytic arc-analytic functions.

\subsection{The Nash case}

In this section $M$ is an affine Nash manifold, i.e. a semialgebraic analytic submanifold of some $\mathbb{R}^{k}$. Functions on $M$ which are analytic and semialgebraic are called Nash functions. They form a ring that we denote by $\mathcal{N}(M)$.

Proposition 3.5. Let $M \subset \mathbb{R}^{k}$ be a Nash manifold and let $S \subset M$ be a semialgebraic set such that no wall of $S$ is simultaneously odd and even. Then $S \backslash \partial_{Z} S$ is Nash principal open, i.e. $S \backslash \partial_{Z} S=\{f>0\}$ with $f \in \mathcal{N}(M)$. In particular, a semialgebraic set is Nash principal open if and only if it is principal open as a global semianalytic set. 
Proof. By Proposition 2.5, there exists $f \in \mathcal{O}(M)$ such that $S \backslash \partial_{Z} S=\{f>0\}$. Let $Y$ be the union of the odd components of $\partial_{Z} S$ of codimension one in $M$. By the proof of Proposition 2.5, we can assume that $f=h p$ where $p \in \mathcal{N}(M)$ is a positive equation for $\partial_{Z} S$ and $h \in \mathcal{O}(M)$ is a generator for the principal ideal $\mathcal{J}(Y) \mathcal{O}(M)$. Let $g \in \mathcal{N}(M)$ be a positive equation of $Y$. There exists $q \in \mathcal{O}(M)$ such that $g=h q$. By the factorization conjecture ([14]), we can find $\phi_{1}, \phi_{2}$ strictly positive analytic functions on $M$ and $\psi_{1}, \psi_{2}$ Nash functions on $M$, such that $\phi_{1} \phi_{2}=1$ and $h=\psi_{1} \phi_{1}$ and $q=\psi_{2} \phi_{2}$. The function $\psi_{1} p$ is Nash on $M$ and $\operatorname{sign} \psi_{1}=\operatorname{sign} h$ on $M$. Then, $S \backslash \partial_{Z} S=\left\{\psi_{1} p>0\right\}$ is Nash principal.

We use this result to study Nash constructible functions. A semialgebraic set $S \subset$ $M$ is arc-symmetric if for each analytic arc $\gamma:]-1,1[\rightarrow M$ such that $\gamma(]-1,0[) \subset S$, we have $\gamma(]-1,1[) \subset S$ (cf. [17]). Note that the walls of a Nash constructible function are semialgebraic and arc-symmetric and coincide with the walls defined in [10].

Lemma 3.6. Let $\varphi: M \rightarrow\{-1,1\}$ be a Nash constructible function whose walls are irreducible as semialgebraic arc-symmetric sets. Then, there is $f \in \mathcal{N}(M)$ such that $\varphi=$ gen $\operatorname{sign} f$.

Proof. We prove that $\varphi^{-1}(1)$ is generically Nash principal. Assume that a wall $W$ of $\varphi^{-1}(1)$ is simultaneously odd and even. This would mean that $\partial_{W} \varphi$ is not generically constant mod 2 on the irreducible arc-symmetric semialgebraic set $W$. So $\partial_{W} \varphi$ would not be generically Nash constructible on $W$ by [10, Prop.1.5]. This would contradict the generic Nash constructibility of $\varphi$, since Nash constructible functions are preserved by the operator $\frac{1}{2} \Lambda([19])$. So no wall of $\varphi^{-1}(1)$ is simultaneously odd and even, and by Proposition 3.5 the proof is done.

Lemma 3.7. Let $M$ be an affine Nash manifold of dimension two. A function $\varphi$ : $M \rightarrow \mathbb{Z}$ is Nash constructible if and only if it is a sum of signs of Nash functions.

Proof. We can copy the proofs of Theorem 2.10 and Theorem 3.3, since the stability index of $\mathcal{N}(M)$ is two, and since an irreducible analytic curve is irreducible as an arc-symmetric set (cf. [17]).

Again, this result is false in general for a Nash manifold of dimension $\geq 3$ or a singular set, see [10, Rk.3.5].

Repeating the proof of Theorem 3.6 in [10] we get:

Corollary 3.8. Let $X \subset \mathbb{R}^{k}$ be a semialgebraic arc-symmetric set of dimension two. A function $\varphi: X \rightarrow \mathbb{Z}$ is Nash constructible if and only if $\varphi$ is a sum of signs of semialgebraic arc-analytic functions on $X$. 


\section{References}

[1] F. Acquistapace, C. Andradas, and F. Broglia, Separation of semialgebraic sets, J. Amer. Math. Soc. 12 (1999), 703-728.

[2] F. Acquistapace and F. Broglia, More about signatures and approximation, Geom. Dedicata 50 (1994), 107-116.

[3] F. Acquistapace, F. Broglia, and E. Fortuna, A separation theorem in dimension 3, Nagoya Math. J. 143 (1996), 171-193.

[4] F. Acquistapace, F. Broglia, and M. P. Vélez, Basicness of semialgebraic sets, Geom. Dedicata 78 (1999), 229-240.

[5] C. Andradas, L. Bröcker, and J. M. Ruiz, Constructible sets in real geometry, Ergebnisse der Mathematik und ihrer Grenzgebiete (3), vol. 33, Springer-Verlag, Berlin, 1996, ISBN 3-54060451-0.

[6] C. Andradas, A. Díaz-Cano, and J. M. Ruiz, The Artin-Lang property for normal real analytic surfaces, J. Reine Angew. Math. 556 (2003), 99-111.

[7] E. Becker and L. Bröcker, On the description of the reduced Witt ring, J. Algebra 52 (1978), $328-346$.

[8] E. Bierstone and P. D. Milman, Arc-analytic functions, Invent. Math. 101 (1990), 411-424.

[9] I. Bonnard, Un critère pour reconaître les fonctions algébriquement constructibles, J. Reine Angew. Math. 526 (2000), 61-88. (French, with English summary)

[10] _ Nash constructible functions, prépublication de l'Université d'Angers, 2001.

[11] F. Broglia and F. Pieroni, Separation of global semianalytic subsets of 2-dimensional analytic manifolds, Pacific J. Math. 214 (2004), 1-16.

[12] A. Castilla, Artin-Lang property for analytic manifolds of dimension two, Math. Z. 217 (1994), $5-14$.

[13] M. Coste and K. Kurdyka, Le discriminant d'un morphisme de variétés algébriques réelles, Topology 37 (1998), 393-399.

[14] M. Coste and M. Shiota, Nash functions on noncompact Nash manifolds, Ann. Sci. École Norm. Sup. (4) 33 (2000), 139-149. (English, with English and French summaries)

[15] A. Díaz-Cano and C. Andradas, Complexity of global semianalytic sets in a real analytic manifold of dimension 2, J. Reine Angew. Math. 534 (2001), 195-208.

[16] M. Kashiwara and P. Schapira, Sheaves on manifolds, Grundlehren der Mathematischen Wissenschaften, vol. 292, Springer-Verlag, Berlin, 1994, ISBN 3-540-51861-4.

[17] K. Kurdyka, Ensembles semi-algébriques symétriques par arcs, Math. Ann. 282 (1988), 445462 .

[18] $\ldots$ An arc-analytic function with nondiscrete singular set, Ann. Polon. Math. 59 (1994) $251-254$.

[19] C. McCrory and A. Parusiński, Algebraically constructible functions, Ann. Sci. École Norm. Sup. (4) 30 (1997), 527-552. (English, with English and French summaries)

[20] C. McCrory and A. Parusiński, Algebraically Constructible Functions: Real Algebra and Topology, available on http://www.maths .univ-rennes1.fr/ raag01/Surveys.html.

[21] A. Parusiński, Subanalytic functions, Trans. Amer. Math. Soc. 344 (1994), 583-595.

[22] A. Parusiński and Z. Szafraniec, On the Euler characteristic of fibres of real polynomial maps, Singularities Symposium - Lojasiewicz 70 (Kraków, 1996; Warsaw, 1996), Banach Center Publ., vol. 44, Polish Acad. Sci., Warsaw, 1998, pp. 175-182.

[23] P. Schapira, Operations on constructible functions, J. Pure Appl. Algebra 72 (1991), 83-93. 